\title{
Reply on "Successful Conservative Management of Hepatic Portal Venous Gas due to Anastomosis Leakage After a Sigmoidectomy"
}

\author{
Seongwoo Hong \\ Department of Surgery, Inje University Seoul Paik Hospital, Seoul, Korea
}

To the Editor,

We appreciate and thank Dr. Carannante et al. [1] for their interest in our work [2] and comments. Our case involved hepatic portal vein gas (HPVG), which developed due to anastomosis leakage after sigmoidectomy. The unique aspect of our case was that HPVG developed as a surgical complication immediately after colon resection. Capolupo et al. [3] reported an HPVG case that developed at an ileoanal anastomosis site constructed 8 years prior and the cause of HPVG was an endoscopic procedure. Li et al. [4] reported HPVG that developed due to colon cancer obstruction and perforation. Their case was not related to surgical complications after colectomy. We suggest that HPVG that develops as a surgical complication after colectomy can be managed conservatively if clinical symptoms and signs are stable.

\section{CONFLICT OF INTEREST}

No potential conflict of interest relevant to this article was reported.

\section{REFERENCES}

1. Carannante F, Capolupo GT, Masciana G, Caricato M. Hepatic portal venous gas and anastomotic leakage. Ann Coloproctol 2020;36:211.

2. Hong I, Hong SW, Chang YG, Lee B, Lee WY, Ohe HJ, et al. Successful conservative management of hepatic portal venous gas due to anastomosis leakage after a sigmoidectomy. Ann Coloproctol 2019;35:282-4.

3. Capolupo GT, Masciana G, Carannante F, Caricato M. Hepatic portal venous gas after colonoscopy: a case report and review. Int J Surg Case Rep 2018;51:54-7.

4. Li Z, Su Y, Wang X, Yan H, Sun M, Shu Z. Hepatic portal venous gas associated with colon cancer: a case report and literature review. Medicine (Baltimore) 2017;96:e9352. 